\title{
Controlled generation of mixed spatial qudits with arbitrary degree of purity
}

\author{
J. J. M. Varga, ${ }^{*}$ S. Ledesma, and C. Iemmi \\ Departamento de Física, Universidad de Buenos Aires, Facultad de Ciencias Exactas y Naturales, Buenos Aires, Argentina \\ and Consejo Nacional de Investigaciones Científicas y Técnicas, Buenos Aires, Argentina \\ L. Rebón \\ Departamento de Física, IFLP, Universidad Nacional de La Plata, C.C. 67, 1900 La Plata, Argentina
}

(Received 5 June 2017; published 8 September 2017)

\begin{abstract}
We propose a method for preparing mixed quantum states of arbitrary dimension $D(D \geqslant 2)$ which are codified in the discretized transverse momentum and position of single photons, once they are sent through an aperture with $D$ slits. Following our previous technique we use a programmable single phase-only spatial light modulator (SLM) to define the aperture and set the complex transmission amplitude of each slit, allowing the independent control of the complex coefficients that define the quantum state. Since these SLMs give us the possibility to dynamically vary the complex coefficients of the state during the measurement time, we can generate not only pure states but also quantum states compatible with a mixture of pure quantum states. Therefore, by using these apertures varying on time according to a probability distribution, we have experimentally obtained $D$-dimensional quantum states with purities that depend on the parameters of the distribution through a clear analytical expression. This fact allows us to easily customize the states to be generated. Moreover, the method offers the possibility of working without changing the optical setup between pure and mixed states, or when the dimensionality of the states is increased. The obtained results show a quite good performance of our method at least up to dimension $D=11$, being the fidelity of the prepared states $F>0.98$ in every case.
\end{abstract}

DOI: 10.1103/PhysRevA.96.032309

\section{INTRODUCTION}

In quantum optics, pure quantum states of single photons have been widely explored, both theoretically and experimentally. They can be generated, controlled, and measured using the several degrees of freedom of a photon, and by means of different techniques [1-3]. However, because of experimental imperfections or interactions with the environment, a quantum system is not in general in a pure state, and only a partial knowledge of its physical state can be obtained. In this way it cannot be described through a well defined vector $|\psi\rangle$ in Hilbert space. For that reason, the most general description of a quantum system is given by a mixture of pure quantum states that can be mathematically expressed by the formalism of the density matrix [4]. In consequence, the progress in the study of quantum systems and their potentialities for practical applications relies on the ability for controlling mixed states, and not only pure states. For instance, the ability for engineering and measuring mixed quantum states allows one to experimentally study how quantum computing algorithms and quantum communication protocols are affected by decoherence $[5,6]$. Besides, beyond the original model for quantum information $[7,8]$, based in unitary gates operating on pure quantum states, alternative models based on mixed quantum states have been developed $[9,10]$. These models also give the possibility to perform some tasks not realizable with a comparable classical system [11-14]. Moreover, as the system is initially in a mixed quantum state, and entanglement is not the required physical resource, they are less restrictive, more robust against noise, and easier to implement than the standard quantum information model.

\footnotetext{
*miguel@df.uba.ar
}

Controllable generation of mixed quantum states has been successfully proposed in earlier works, mainly, using the polarization degree of freedom to codify the state [15-18]. While these methods are relatively simple to implement, they only allow the realization of two-level systems. Otherwise, higher dimensional quantum states, namely, qudits ( $D$-level quantum systems), increase the quantum complexity without increasing the number of particles involved. For instance, systems of dimension $D=2^{N}$ can be used to simulate a composite system of $N$ qubits [19]. For quantum communication protocols, $D$-dimensional quantum channels show higher capacity, and provide better security against an eavesdropper [20-22]. Moreover, multilevel information carriers are crucial to reduce the number of gates required in the circuits for quantum computing [23].

Among the feasible degrees of freedom for encoding high-dimensional quantum systems [24-27], the discretized transverse momentum-position of single photons have attracted particular interest. They have proven useful for several application such as quantum information protocols $[28,29]$, quantum games [30], quantum algorithms [31], and quantum key distribution [32]. The encoding process is achieved by sending the photons through an aperture with $D$ slits, which sets the qudits dimension [33]. More sophisticated methods to generate these so-called spatial qudits, take advantage of liquid crystal displays (LCDs) as programmable spatial light modulators (SLMs). These programmable optical devices can be used to define a set of independent $D$ slits with complex transmission. In this way, it is possible to produce and measure arbitrary pure qudits without any extra physical alignment of the optical components [19,34-36].

Recently, Lemos et al. [37] have characterized the action of an SLM as a noisy quantum channel acting on a polarization qubit, and they used it for implementing a phase flip channel 
with a controllable degree of decoherence. In Ref. [38] Marques et al. extended the use of the SLMs to simulate the open dynamics of a $D$-dimensional quantum system by using films instead of images.

In this paper, we present a method to generate arbitrary spatial mixed states of $D$ dimensions $(D \geqslant 2)$, which is based on the techniques developed in our previous works $[35,36]$. We have extended these techniques to consider $D$ slits with a variable complex transmission. The use of a programmable SLM makes it possible to dynamically modify the complex transmission, in order to obtain a mixed qudit state by averaging the sample over time.

The paper is organized as follows: In Sec. II we give the mathematical description of a single-photon state when it is sent trough an aperture with a time-varying transmission function. By considering that we can vary the relative phase values of the complex transmission following a uniform probability distribution, we have derived simple analytical expressions, which show the dependence between the distribution widths and the purity of the state, for any dimension $D$. From these expressions it is possible to obtain any degree of purity by continuously varying the the distribution widths, which allows us to use the same method for preparing pure and mixed states. In Sec. III A it is described the experimental setup and it is explained how a first SLM is addressed to generate the states, while a second SLM is employed to encode the measurement bases used to perform the tomographic reconstruction of the system. In Sec. III B it is reported a first experiment, consisting in the generation and measurement of pure qudit states. It was carried on in order to test the setup and the proposed methods. Afterwards, in Sec. III C, we implement the variable transmission function for generating mixed states with different degrees of purity in dimensions $D=2,3,7$, and 11. Finally, the results are presented in Sec. IV and discussed before going into the conclusions.

\section{FORMALISM}

Let us start by considering the generation of a spatial qudit in a pure state. A paraxial and monochromatic single-photon field is transmitted through an aperture described by a complex transmission function $A(\mathbf{x})$. Assuming an initial pure state $|\psi\rangle$, it is transformed as

$$
|\psi\rangle=\int d \mathbf{x} \psi(\mathbf{x})|1 \mathbf{x}\rangle \stackrel{A(\mathbf{x})}{\Longrightarrow} \int d \mathbf{x} \psi(\mathbf{x}) A(\mathbf{x})|1 \mathbf{x}\rangle,
$$

where $\mathbf{x}=(x, y)$ is the transverse position coordinate and $\psi(\mathbf{x})$ is the normalized transverse probability amplitude for this state, i.e., $\int d \mathbf{x}|\psi(\mathbf{x})|^{2}=1$.

We are interested in generating an incoherent mixture of pure states by varying the transmission function of the aperture over time. So, let us consider that $A(\mathbf{x}, t)$ is an array of $D \geqslant 2$ rectangular slits of width $2 a$, period $d$, and length $L(\gg a, d)$, where each slit $\ell$ has a transmission amplitude $\beta_{\ell}(t)$ :

$$
A(\mathbf{x}) \rightarrow A(\mathbf{x}, t)=\operatorname{rect}\left(\frac{x}{L}\right) \sum_{\ell=0}^{D-1} \beta_{\ell}(t) \operatorname{rect}\left(\frac{y-\eta_{\ell} d}{2 a}\right)
$$

with $\eta_{\ell}=\ell+(D-1) / 2$.
Thus, instantaneously, at any time $t$ a pure state $|\psi(t)\rangle$ is obtained, whereas in a finite period of time $\Delta t$, an ensemble of these pure states is created. In consequence, the result after a measurement process is the ensemble average over the integration time $T$, whose statistics corresponds to a mixed state described by the density matrix [4] $\rho$ :

$$
\begin{aligned}
\rho & =\frac{1}{T} \int_{0}^{T} d t|\psi(t)\rangle\langle\psi(t)| \\
& =\int d \mathbf{x} \int d \mathbf{x}^{\prime} \rho\left(\mathbf{x}, \mathbf{x}^{\prime}\right)|1 \mathbf{x}\rangle\left\langle 1 \mathbf{x}^{\prime}\right|,
\end{aligned}
$$

where $\rho\left(\mathbf{x}, \mathbf{x}^{\prime}\right) \equiv \psi(\mathbf{x}) \psi^{*}\left(\mathbf{x}^{\prime}\right) \frac{1}{T} \int_{0}^{T} d t A(\mathbf{x}, t) A^{*}(\mathbf{x}, t)$. Hence, the state of the photon in Eq. (3) can be written as

$$
\rho=\sum_{\ell, \ell^{\prime}=0}^{D-1} \tilde{c}_{\ell, \ell^{\prime}}|\ell\rangle\left\langle\ell^{\prime}\right|
$$

where $|\ell\rangle$ denotes the state of the photon passing through the slit $\ell$ [26]. The states $|\ell\rangle$ satisfy the condition $\left\langle\ell \mid \ell^{\prime}\right\rangle=\delta_{l l^{\prime}}$, and they are used to define the logical base for spatial qudits. The quantum state of the system is determined by the coefficients $\tilde{c}_{\ell, \ell^{\prime}}=\frac{1}{T} \int_{0}^{T} d t \beta_{\ell}(t) \beta_{\ell^{\prime}}^{*}(t)$, which carry the information codified in the transfer function $A(\mathbf{x}, t)$. In principle, given that in the most general case the transmission amplitudes $\beta_{\ell}(t)$ are complex values, we could introduce the time dependence either in the modulus $\left|\beta_{\ell}(t)\right|$, or in the $\operatorname{argument} \operatorname{Arg}\left[\beta_{\ell}(t)\right]$, and even in both. However, as it is well known, phase information plays a more important role than the real amplitude in signal processing [39] so we can get full control of the state by varying only the phases (see Sec. III C for a complete discussion). Then, for a time-dependent phase $\phi_{\ell}(t)$, the transmission for the slit $\ell$ is written as $\beta_{\ell}(t)=\beta_{\ell} e^{i \phi_{\ell}(t)}$, and the complex coefficients in the mixture in Eq. (4) are given by the expression

$$
\tilde{c}_{\ell, \ell^{\prime}}=\left(\beta_{\ell} \beta_{\ell^{\prime}} / \sqrt{\sum_{j=0}^{D-1} \beta_{j}^{2}}\right) c_{\ell, \ell^{\prime}}
$$

with

$$
c_{\ell, \ell^{\prime}}=\frac{1}{T} \int_{0}^{T} d t e^{i \phi_{\ell}(t)} e^{-i \phi_{\ell^{\prime}}(t)} .
$$

To define this state we have proposed that the phase of each slit varies according to a probabilistic distribution. If the time $T$ is much longer than the characteristic time where the phase varies, the integration in the time domain can be replaced by an integration in the phase domain $\Omega$. In fact, we can assume that for a period of time long enough, $\phi_{\ell}(t)$ reaches all its possible values with a frequency of occurrence given by a probability distribution $f\left(\alpha_{\ell}\right)\left(\alpha_{\ell} \in \Omega\right)$. In addition, if the phase of each slit varies independently of the other ones, the joint probability distribution is obtained as

$$
f(\alpha) \equiv f\left(\alpha_{0}, \alpha_{1}, \ldots, \alpha_{D-1}\right)=f\left(\alpha_{0}\right) f\left(\alpha_{1}\right) \cdots f\left(\alpha_{D-1}\right) .
$$

According to this scheme, the complex coefficients in Eq. (6) turn into

$$
c_{\ell, \ell^{\prime}}=\int d \alpha f(\alpha) e^{i \alpha_{\ell}} e^{-i \alpha_{\ell^{\prime}}}
$$


From this expression we directly obtain $c_{\ell, \ell}=1, \forall \ell=$ $0,1, \ldots, D-1$ and $c_{\ell, \ell^{\prime}}=c_{\ell^{\prime}, \ell}^{*}, \forall \ell, \ell^{\prime}=0,1, \ldots, D-1$, implying that the diagonal elements of the density matrix [Eq. (4)], which denote the probabilities to find the system in one of the (pure) quantum states $|\ell\rangle$, are real-valued coefficients in the interval $[0,1]$, and as expected, the density matrix is Hermitian $\left(\rho^{\dagger}=\rho\right)$.

As only the relative phases (but not the absolute values) in the linear combination that define the quantum state are relevant, we have (indistinctly) fixed the phase value of one of the slits $|0\rangle$ to be $\phi_{0}=0$. Then, the corresponding probability distribution in Eq. (7) is the Dirac $\delta$ function $\delta\left(\alpha_{0}\right)$. Besides, we have assumed that $f\left(\alpha_{\ell}\right)$ is a uniform distribution of width $\Delta_{\ell}$ and centered in $\alpha_{\ell}=\phi_{\ell}, \forall \ell=1, \ldots, D-1$. In this way, the joint probability distribution is

$$
\begin{aligned}
f(\alpha) & =\mathcal{N} \delta\left(\alpha_{0}\right) \prod_{\ell=1}^{D-1} \operatorname{rect}\left(\frac{\alpha_{\ell}-\phi_{\ell}}{\Delta_{\ell}}\right), \\
\mathcal{N} & =\frac{1}{\prod_{j=1}^{D-1} \Delta_{j}} .
\end{aligned}
$$

Therefore, the statistical mixture $\rho$ which describe the state of the transmitted photon, will be completely determined by the real amplitudes $\beta_{\ell}$, the phases $\phi_{\ell}$, and the distribution widths $\Delta_{\ell}$, which can be completely and independently controlled in our experimental setup (see Sec. III A). Even more, it is straightforward to obtain the purity of the state, $P(\rho) \equiv \operatorname{Tr}\left(\rho^{2}\right)$ :

$$
\begin{aligned}
P(\rho)= & \mathcal{Z}^{2} \sum_{i=0}^{D-1} \beta_{i}^{4} \\
& +2 \mathcal{Z}^{2} \sum_{i=0}^{D-2} \prod_{j>i}^{D-1} \beta_{i}^{2} \beta_{j}^{2} \operatorname{sinc}\left(\frac{\Delta_{i}}{2}\right)^{2} \operatorname{sinc}\left(\frac{\Delta_{j}}{2}\right)^{2},
\end{aligned}
$$

being the normalization constant $\mathcal{Z}=\left(\sum_{i=0}^{D-1} \beta_{i}^{2}\right)^{-1}$. From this equation [Eq. (10)] it becomes clear how to generate a qudit state with an arbitrary purity, by setting up the experimental parameters. In particular, the maximal mixed state $\left[P(\rho)=\frac{1}{D}\right]$ is obtained when the real coefficients $\beta_{\ell}$ all have the same value, and the phases can reach any value between 0 and $2 \pi$ with the same probability, i.e., $\Delta_{\ell}=2 \pi, \forall \ell=1, \ldots, D-1$. On the other hand, if $\Delta_{\ell} \rightarrow 0, \forall \ell=1, \ldots, D-1$, i.e., when the phase of each slit remains constant over the time $T$, the terms $\operatorname{sinc}\left(\frac{\Delta_{\ell}}{2}\right)$ are equal to 1 . In such a case, the purity of the state tends to 1 , as expected for a pure quantum state. Thus, the scheme discussed here is reduced to the previous ones presented in Refs. [35] and [36] for preparing arbitrary pure spatial qudits.

Let us consider as an example the preparation of qubit states. Because of their simplicity, they are helpful to understand the general behavior of the scheme. In this case $(D=2)$ we explicitly obtain

$$
c_{01}=\frac{1}{\Delta_{1}} \int_{\phi_{1}-\frac{\Delta_{1}}{2}}^{\phi_{1}+\frac{\Delta_{1}}{2}} d \alpha_{1} e^{-i \alpha_{1}}=e^{-i \phi_{1}} \operatorname{sinc}\left(\frac{\Delta_{1}}{2}\right) .
$$

The diagonal coefficients of the density matrix are independent of the phase probability distribution, since as was mentioned

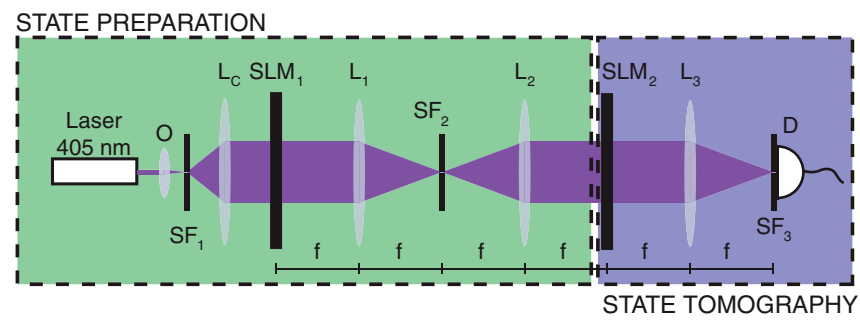

FIG. 1. Experimental setup. $\mathrm{O}$ is an expansor, $\mathrm{SF}_{\mathrm{i}}$ are spatial filters, $L_{i}$ are lenses with a focal distance $f, \mathrm{SLM}_{\mathrm{i}}$ are spatial light modulators, and $\mathrm{D}$ is a single pixel detector.

before $c_{\ell, \ell}=1$, while the rest of coefficients can be obtained by complex conjugation. Therefore, these states are described by the density matrix

$$
\rho=\frac{1}{\beta_{0}^{2}+\beta_{1}^{2}}\left(\begin{array}{cc}
\beta_{0}^{2} & \beta_{0} \beta_{1} e^{-i \phi_{1}} \operatorname{sinc}\left(\frac{\Delta_{1}}{2}\right) \\
\beta_{0} \beta_{1} e^{i \phi_{1}} \operatorname{sinc}\left(\frac{\Delta_{1}}{2}\right) & \beta_{1}^{2}
\end{array}\right) .
$$

They have a purity given by

$$
P(\rho)=\frac{\beta_{0}^{4}+\beta_{1}^{4}+2 \beta_{0}^{2} \beta_{1}^{2} \operatorname{sinc}^{2}\left(\frac{\Delta_{1}}{2}\right)}{\left(\beta_{0}^{2}+\beta_{1}^{2}\right)^{2}},
$$

and any degree of purity can be achieved by controlling the relation $\beta_{1} / \beta_{0}$, and $\Delta_{1}$.

In the next section we described our technique developed for implementing these concepts, and illustrate with the generation of mixed states in different dimensions $D$.

\section{EXPERIMENTAL IMPLEMENTATION}

\section{A. Experimental setup}

The experimental setup used for the generation and reconstruction of the spatial qudit states is shown, schematically, in Fig. 1. The first part consists of a $4 f$ optical system with a spatial filter in the Fourier plane.

A $405 \mathrm{~nm}$ laser diode beam is expanded, filtered, and collimated in order to illuminate the spatial light modulator $\mathrm{SLM}_{1}$ with a planar wave with approximately constant phase and amplitude distribution over the region of interest. This modulator is used to represent the spatial qudit $|\psi\rangle$ according to the techniques described in $[35,36]$. These methods allow us to generate pure spatial qudits with arbitrary complex coefficients by using only one pure phase modulator. The coefficient modulus $\beta_{\ell}$ (see Sec. II) is given by the phase modulation of the diffraction gratings displayed on each slit region. The argument $\phi_{\ell}$ can be defined either by adding a constant phase value [35] or by means of a lateral displacement of the gratings [36]. Both methods have a good performance, the latter one being developed to reduce the effects of the phase fluctuations present in modern liquid crystal on silicon (LCoS) displays [40]. In particular, the phase modulators used in our experiment are conformed by a Sony liquid crystal television panel LCTV model LCX012BL in combination with polarizers and wave plates that provide the adequate state of light polarization to reach a phase modulation near $2 \pi$ at $405 \mathrm{~nm}[41,42]$. As this device is free of phase fluctuations the first codification method was implemented given that it avoids the phase quantization required in the second scheme. 
The spatial filter $\mathrm{SF}_{2}$ is used to select the first orders diffracted by the mentioned gratings in such a way that on the back focal plane of lens $L_{2}$ is obtained the complex distribution that represents the spatial qudit.

On the same plane (which is also coincident with the front focal plane of $\mathrm{L}_{3}$ ) is placed the second modulator $\mathrm{SLM}_{2}$ on which are represented the reconstruction bases $\left|\psi_{m}^{(\alpha)}\right\rangle$ used to implement the quantum state tomography process [19]. These bases are also displayed as slits and its complex amplitudes are codified by following the previously described method. The measurements that allow characterizing the quantum state are performed by means of a single pixel detector placed at the back focal plane of $\mathrm{L}_{3}$ and a spatial filter $\mathrm{SF}_{3}$ used to select the center of the interference pattern produced by the slits.

It is worth to mention that the proposed architecture performs the exact Fourier transform at each stage and avoids the introduction of spurious phases through the propagation process.

Finally, we point out that a single-photon implementation of the method could be carried out by using exactly the same optical setup except that the laser should be attenuated by means of density filters. Alternatively, one could use a singlephoton source based, for example, on a spontaneous parametric down conversion (SPDC) process in a nonlinear crystal. In any case, a single-photon-detection module should be used in the detection stage.

\section{B. Generation of pure states}

In order to test the implementation of the encoding method in our optical setup and optimize the alignment process we started by preparing and reconstructing pure quantum states. The generation of pure states is achieved by representing the state $|\psi\rangle$ on the $\mathrm{SLM}_{1}$, as we explained in Sec. III A. The tomographic process is carried out by means of projective measurements that allow reconstructing the density matrix $\rho$ in Eq. (4). We represent the reconstruction basis $\left|\psi_{m}^{(\alpha)}\right\rangle$ on the $\mathrm{SLM}_{2}$ and take the number of counts in the center of the Fourier plane as the value of the projection $p_{\alpha_{m}}=\left|\left\langle\psi_{m}^{(\alpha)} \mid \psi\right\rangle\right|^{2}$. We use a mutually unbiased basis, which implies to perform $D(D+1)$ projections to finally reconstruct the density matrix as [43]

$$
\rho=\sum_{\alpha=1}^{D+1} \sum_{m=1}^{D} p_{\alpha_{m}}\left|\psi_{m}^{(\alpha)}\right\rangle\left\langle\psi_{m}^{(\alpha)}\right|-I
$$

To quantify the quality of the whole experiment we used the fidelity $F \equiv \operatorname{Tr}(\sqrt{\sqrt{\varrho} \rho \sqrt{\varrho}})$, between the state intended to be prepared, $\varrho$, and the density matrix of the state actually prepared, $\rho$ [44]. Ideally, it is desirable to have $F=1$.

We have tested the system for different Hilbert space dimensions with excellent results. As an example, the reconstruction results obtained for $D=11$ are shown in Fig. 2. To this end we have generated 500 pure states $|\psi\rangle=\sum e^{i \phi_{\ell}}|l\rangle$ with an arbitrary phase $\phi_{\ell}$ uniformly distributed between 0 and $2 \pi$. The mean fidelity is $\bar{F}=0.992$ with standard deviation $\sigma=0.003$. The system proved to be reliable for the generation and reconstruction of pure qudits in different dimensions.

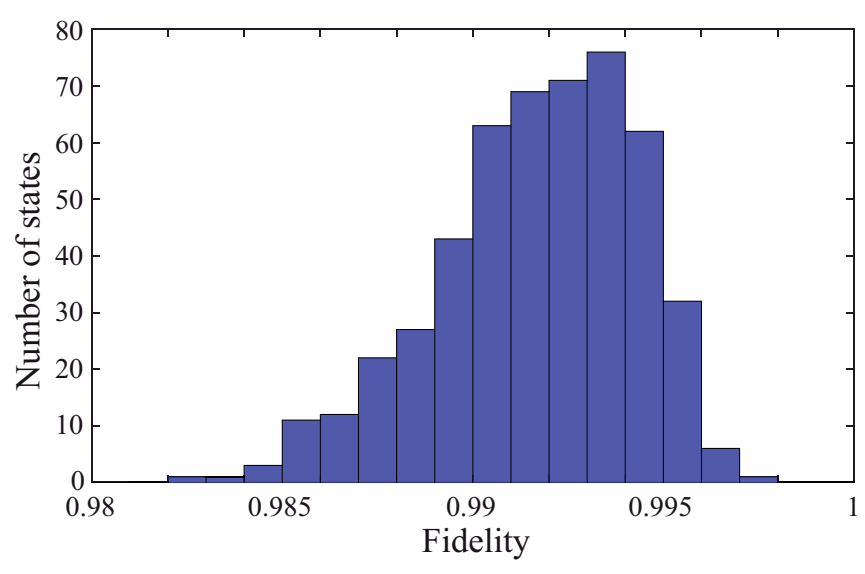

FIG. 2. Fidelity occurrence for qudits states with $D=11$. There are represented 500 arbitrary states $|\psi\rangle=\sum_{\ell=0}^{D-1} e^{i \phi_{\ell}}|\ell\rangle$. The mean fidelity is $\bar{F}=0.992$ and the standard deviation is $\sigma=0.003$.

\section{Generation of mixed states}

The mixed states generation is achieved by means a statistical mixture of pure states $|\psi\rangle$. This can be performed by varying the modulus $\left|\beta_{\ell}(t)\right|$ and/or phases $\phi_{\ell}(t)$ of the states represented on $\mathrm{SLM}_{1}$ while the measurement process is carried on. As previously mentioned, in Sec. II, in general, many of the important features of a signal are preserved when only the phase is retained regardless of the amplitude [39]. In order to verify this assertion in our case, we studied, first by numerical simulation, the effect of varying separately these magnitudes. We started by keeping constant the amplitudes and changing the phases with a uniform probability distribution centered on a mean phase value $\phi_{\ell}$, and with a width $\Delta_{\ell}$. The purity of the states are determined by the width $\Delta_{\ell}$ as is stated in Eq. (10). The highest incoherence is achieved when $\Delta_{\ell}=2 \pi$ for each slit, and narrower widths lead to greater coherence between slits. As pure states are added to the mixture, purity converges to a steady value. As an example, in Fig. 3 it is shown the purity evolution as a function of the number of pure states used to generate a mixed state of dimension $D=2$. The evolution is

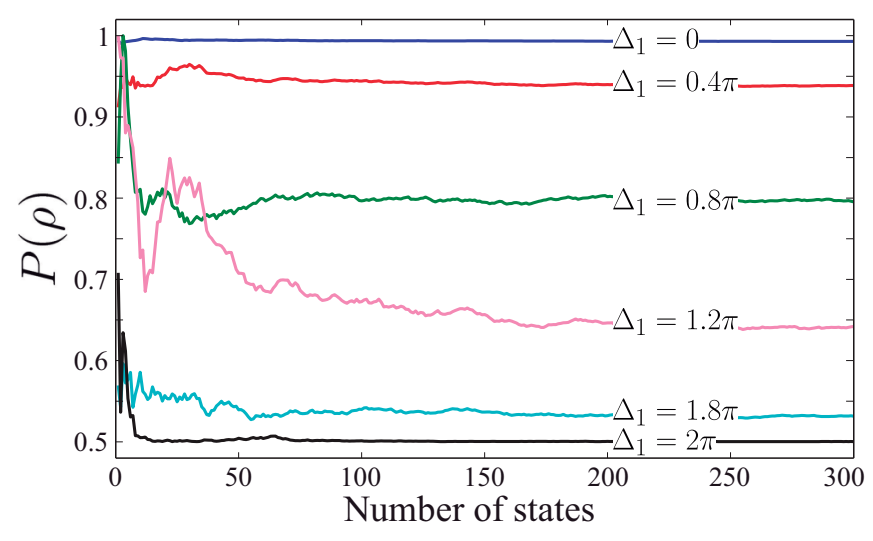

FIG. 3. Purity evolution for qubits as a function of the number of states composing the statistical mixture, and different probability distribution widths $\Delta_{1}$. We note that after 250 pure states were used to generate the mixture, the purity behavior stabilizes and it achieves its final value. 


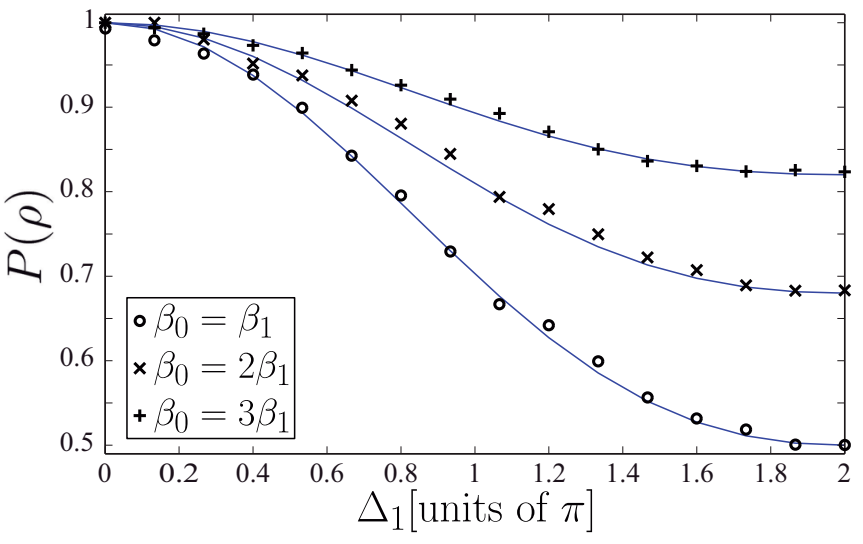

FIG. 4. Purity of qubits as a function of the probability distribution width $\Delta_{1}$ for different relative amplitudes $\beta_{0}$ and $\beta_{1}$. The solid line represents the theoretical values according to Eq. (12).

depicted for different width distributions. We can observe that there is a stabilization afterwards 250 pure states were used to generate the mixture. A similar behavior was observed for higher dimensions $D$ (see Supplemental Material [45]).

Following the same technique for generating mixed states, we also tested the purity evolution of the states by varying the real amplitudes $\beta_{\ell}$, instead of the phases $\phi_{\ell}$. We have observed that the convergence to a steady purity value is obtained after adding (at least) 500 pure states in the mixture. Besides, independently of which distribution width $\Delta_{\ell}$ is considered, it is not possible to achieve the lowest purity value. Summarizing, phase variation results in the best option in order to generate mixed states.

\section{RESULTS}

In this section are presented and analyzed the results obtained for mixed states ranging from dimension $D=2$ to $D=11$. Let us start with the mixed qubits case. We have generated states with three different relative amplitudes of the two slits $\left(\beta_{0}\right.$ and $\left.\beta_{1}\right)$ and diverse width distributions of the phase variations $\left(\Delta_{1}\right)$. The purity of the states, $P(\rho)$, as a function of these magnitudes is shown in Fig. 4. In every case the experimental values of purity matches very well with the theoretical behavior described by Eq. (12).
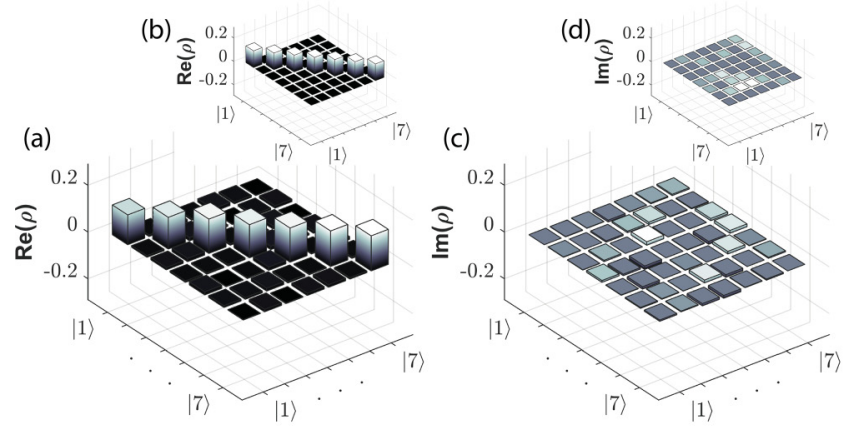

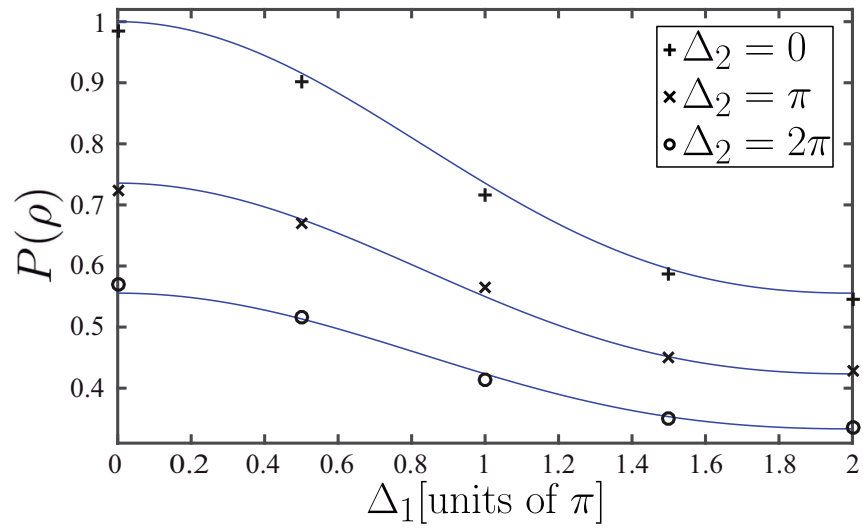

FIG. 5. Purity of $D=3$ qudits as a function of the probability distribution width $\Delta_{1}$ for different fixed widths $\Delta_{2}$. The relative amplitudes are $\beta_{0}=\beta_{1}=\beta_{2}$. The solid line represents the theoretical values according to Eq. (10).

For $\Delta_{1}=2 \pi$ in the case of $\beta_{0}=\beta_{1}$, represented with circles, it is possible to reach the lowest purity for qudits, $P(\rho)=\frac{1}{2}$. However, in the cases where $\beta_{0}=2 \beta_{1}$ (crosses) and $\beta_{0}=3 \beta_{1}$ (squares) the lowest purity obtained is higher than in the first case. In fact, the value of purity defined by Eq. (12) is a function of $\beta_{0}, \beta_{1}$, and $\Delta_{1}$, and the lowest purity achievable is obtained for $\beta_{0}=\beta_{1}$ and $\Delta_{1}=2 \pi$.

In the case of qutrits $(D=3)$, we have generated several mixed states. A particular situation is illustrated in Fig. 5. It shows the purity of these states as a function of the probability width $\Delta_{1}$ of the second slit for different widths $\Delta_{2}$ fixed on the third slit. The relative amplitudes between slits are $\beta_{0}=\beta_{1}=$ $\beta_{2}$. In the case of $\Delta_{2}=2 \pi$ (circles), the lowest possible purity $P(\rho)=\frac{1}{3}$ is reached for $\Delta_{1}=2 \pi$. Lower values of $\Delta_{2}$, in this case $\Delta_{2}=\pi$ (crosses) and $\Delta_{2}=0$ (plus signs), leads to higher purities. The situation becomes trivial for $\Delta_{1}=\Delta_{2}=0$ when a pure state $[P(\rho)=1]$ is obtained.

For $D=7$ the density matrices corresponding to two different mixed states are shown in Fig. 6. For both states the amplitudes of the slits are equal, i.e., $\beta_{0}=\beta_{1}=\cdots=\beta_{6}$. On the left side it is shown the case of lowest coherence between slits, obtained when $\Delta_{\ell}=2 \pi$ for every slit. It can be seen that the diagonal elements on the real part (the system populations)

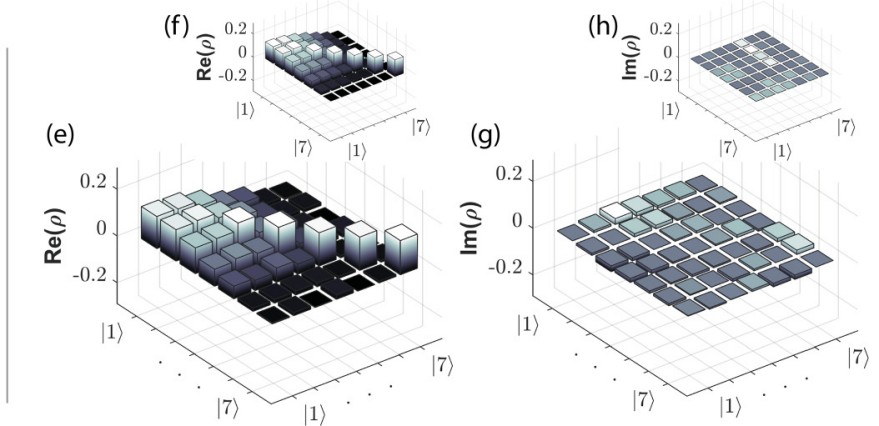

FIG. 6. Density matrices of mixed states in a $D=7$ Hilbert space. In the case of $\Delta_{\ell}=2 \pi$, panels (a) and (c) represent, respectively, the real and imaginary parts reconstructed after a mixture of 250 pure states. (b) and (d) are the corresponding theoretical matrices. When $\Delta_{\ell}=\frac{2 \pi}{7} \ell$, panels (e) and (g) show the real and imaginary parts, obtained after 250 pure states were mixed, while (f) and (h) are the corresponding theoretical matrices. 
(a)

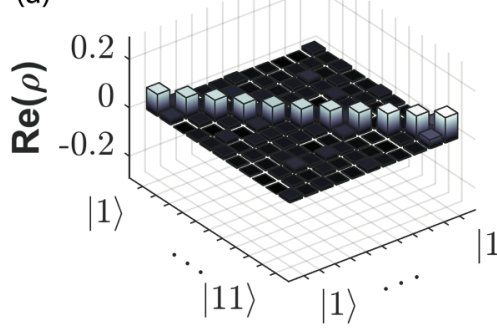

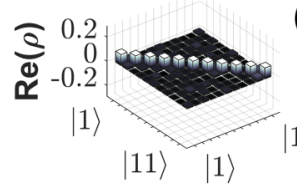

(a)

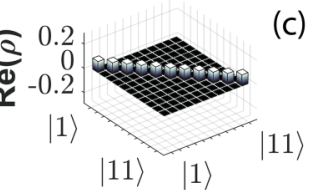

(b)

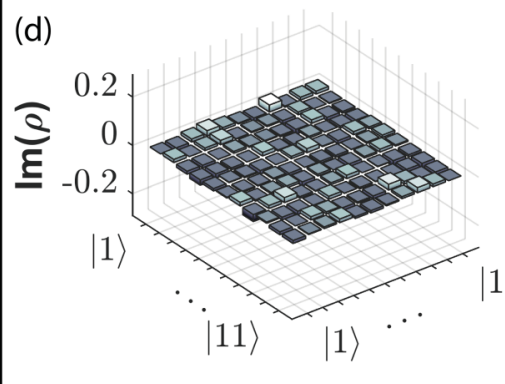

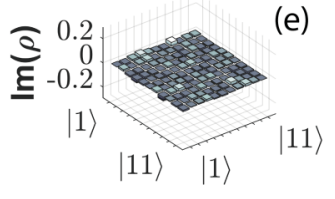

(f)

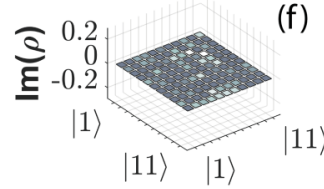

FIG. 7. Density matrices for a $D=11$ mixed state, when $\Delta_{\ell}=2 \pi$. Panels (a) and (d) are, respectively, the real and imaginary parts reconstructed from the mixture of 250 pure states. Correspondingly, (b) and (e) are the numerically simulated results obtained from the mixture of the same 250 pure states. Panels (c) and (f) represent the theoretical matrices.

are equal and different from zero, while the off-diagonal elements (the system coherences) are null. On the right side is shown the density matrix of a state whose slit coherences are governed by probability distribution widths that follow a linear dependence with the slit label $\ell$, that is, $\Delta_{\ell}=\frac{2 \pi}{7} \ell$. It can be noted that the system populations remain equal, like in the previous case, but the system coherences decrease as the slit label increases. These examples show that, by means of the proposed method, it is possible to modify the coherence between the slits in an arbitrary way.

In the case $D=11$ we present two mixed states with different coherences among slits. Figure 7 shows the real (left) and imaginary (right) parts of an incoherent state, and thus, with minimal purity. All the relative amplitudes are equal and the phase distribution widths are $\Delta_{\ell}=2 \pi$. Figures 7(a) and 7(d) show the real and imaginary part of the reconstructed density matrix by mixing 250 pure states. Figures 7(b) and 7(e) show simulated results using the same 250 states, and Figs. 7(c) and 7(f) the theoretical density matrix. The fidelity between experimental and simulated density matrices is reported as $F=0.9886$. The reported purities are $P(\rho)_{\exp }=0.1201$ and $P(\rho)_{\text {sim }}=0.1177$ for experimental and simulated density matrices, respectively. In this case the lowest purity for a $D=11$ state is $P(\rho)_{\text {theo }}=\frac{1}{11} \sim 0.0909$. We note that the agreement between experimental and simulated results are excellent. The theoretical value corresponds to a mixture of infinite pure states, and this is the reason for not having reached the maximum incoherence. In the Supplemental Material [45] it is shown a dynamical evolution from the initial pure state to the final mixed state.

Same as in the case $D=7$, for $D=11$ we have generated a mixed state with arbitrary coherences among slits. Figure 8 shows the real (left) and imaginary (right) parts of this mixed state. In this case the phase distribution width is given by $\Delta_{\ell}=\frac{2 \pi}{11}(11-\ell)$. Figures $8(\mathrm{a})$ and $8(\mathrm{~d})$ show the real and imaginary part of the reconstructed density matrix by mixing 250 pure states. Figures 8 (b) and $8(\mathrm{e})$ show simulated results using the same 250 states, and Figs. $8(\mathrm{c})$ and $8(\mathrm{f})$ is the theoretical density matrix. The fidelity between experimental and simulated density matrices is $F=0.9955$. The reported purities are $P(\rho)_{\text {exp }}=0.3152$ and $P(\rho)_{\text {sim }}=0.2870$ for experimental and simulated density matrices, respectively. In this case the lowest purity for a $D=11$ state is $P(\rho)_{\text {theo }}=0.2670$. Additionally a demonstration of the convergence is shown in the Supplemental Material [45].

\section{CONCLUSIONS}

We have presented a method for the controlled generation of mixed spatial qudits with an arbitrary degree of purity. The state generation is achieved by a succession of random pure qudits according to a preset probability distribution. We have experimentally showed the viability of the method for qudits from dimension $D=2$ up to $D=11$. The excellent agreement between experimental, simulated, and theoretical results demonstrates the feasibility of the method to easily control the coherence between each pair of slits, that allow
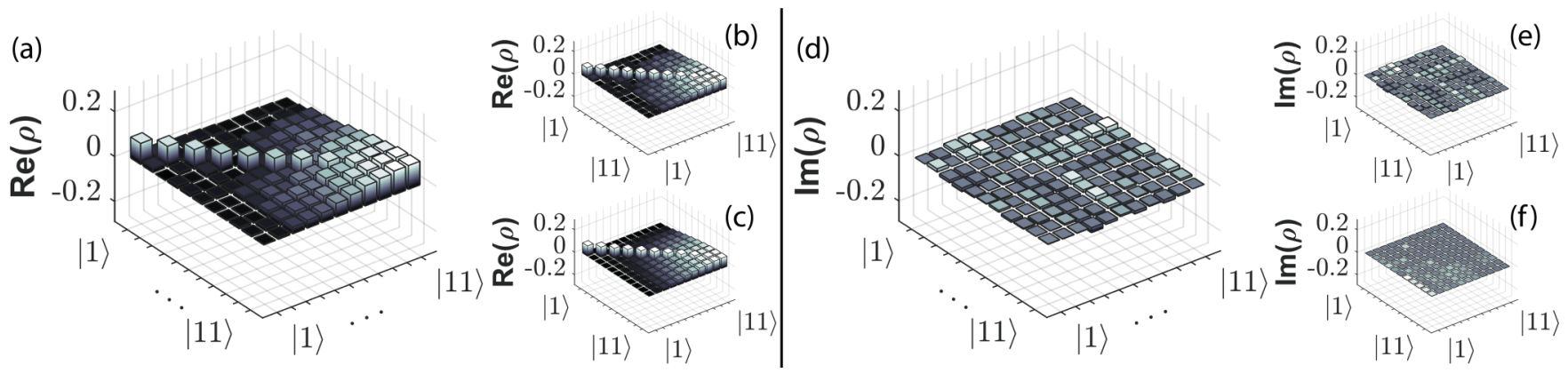

FIG. 8. Density matrices for a $D=11$ mixed state, when $\Delta_{\ell}=\frac{2 \pi}{11}(11-\ell)$. Panels (a) and (d) are, respectively, the real and imaginary parts reconstructed from the mixture of 250 pure states. Correspondingly, (b) and (e) are the numerically simulated results obtained from the mixture of the same 250 pure states. Panels (c) and (f) represent the theoretical matrices. 
us to engineer the state. The method can be extended for the generation of composite systems with controllable degrees of entanglement or mixedness. Besides, it can be used to study the evolution of the system under specific dynamics since the same technique permits to vary, independently, the phases and/or the real amplitude of the slits, i.e., the complex coefficients that define the quantum state of the system.

\section{ACKNOWLEDGMENTS}

This work was supported by UBACyT 20020130100727BA, CONICET PIP 11220150100475CO, and ANPCYT PICT 2014/2432. The authors give thanks to M. A. Solís-Prosser for a fruitful discussion about the postmeasurement density matrix treatment.
[1] P. G. Kwiat, K. Mattle, H. Weinfurter, A. Zeilinger, A. V. Sergienko, and Y. Shih, New High-Intensity Source of Polarization-Entangled Photon Pairs, Phys. Rev. Lett. 75, 4337 (1995).

[2] A. G. White, D. F. V. James, P. H. Eberhard, and P. G. Kwiat, Nonmaximally Entangled States: Production, Characterization, and Utilization, Phys. Rev. Lett. 83, 3103 (1999).

[3] P. Kok and B. W. Lovett, Introduction to Optical Quantum Information Processing (Cambridge University Press, New York, 2010).

[4] U. Fano, Description of states in quantum mechanics by density matrix and operator techniques, Rev. Mod. Phys. 29, 74 (1957).

[5] J. P. Paz and W. H. Zurek, Environment-Induced Decoherence and the Transition from Quantum to Classical (Springer, Berlin, Heidelberg, 2001), pp. 533-614.

[6] V. Kendon, Decoherence in quantum walks-a review, Math Structures Comp. Sci. 17, 1169 (2007).

[7] M. A. Nielsen and I. L. Chuang, Quantum Computation and Quantum Information (Cambridge University Press, Cambridge, 2000).

[8] G. Jaeger, Quantum Information (Springer, Boston, 2007).

[9] E. Knill and R. Laflamme, Power of One Bit of Quantum Information, Phys. Rev. Lett. 81, 5672 (1998).

[10] A. Datta, A. Shaji, and C. M. Caves, Quantum Discord and the Power of One Qubit, Phys. Rev. Lett. 100, 050502 (2008).

[11] D. A. Meyer, Sophisticated Quantum Search without Entanglement, Phys. Rev. Lett. 85, 2014 (2000).

[12] L. Roa, J. C. Retamal, and M. Alid-Vaccarezza, Dissonance is Required for Assisted Optimal State Discrimination, Phys. Rev. Lett. 107, 080401 (2011).

[13] B. Dakić, Y. O. Lipp, X. Ma, M. Ringbauer, S. Kropatschek, S. Barz, T. Paterek, V. Vedral, A. Zeilinger, Č. Brukner et al., Quantum discord as resource for remote state preparation, Nat. Phys. 8, 666 (2012).

[14] M. R. Frey, K. Gerlach, and M. Hotta, Quantum energy teleportation between spin particles in a Gibbs state, J. Phys. A: Math. Theor. 46, 455304 (2013).

[15] S.-Y. Baek and Y.-H. Kim, Preparation and tomographic reconstruction of an arbitrary single-photon path qubit, Phys. Lett. A 375, 3834 (2011).

[16] B. P. Lanyon, M. Barbieri, M. P. Almeida, and A. G. White, Experimental Quantum Computing without Entanglement, Phys. Rev. Lett. 101, 200501 (2008).

[17] J. Dai, Y. L. Len, Y. S. Teo, L. A. Krivitsky, and B.-G. Englert, Controllable generation of mixed two-photon states, New J. Phys. 15, 063011 (2013).

[18] L. Rebón, R. Rossignoli, J. J. M. Varga, N. Gigena, N. Canosa, C. Iemmi, and S. Ledesma, Conditional purity and quantum correlation measures in two qubit mixed states, J. Phys. B 49, 215501 (2016).

[19] G. Lima, L. Neves, R. Guzmán, E. S. Gómez, W. A. T. Nogueira, A. Delgado, A. Vargas, and C. Saavedra, Experimental quantum tomography of photonic qudits via mutually unbiased basis, Opt. Express 19, 3542 (2011).

[20] H. Bechmann-Pasquinucci and W. Tittel, Quantum cryptography using larger alphabets, Phys. Rev. A 61, 062308 (2000).

[21] M. Bourennane, A. Karlsson, G. Bjrk, N. Gisin, and N. J. Cerf, Quantum key distribution using multilevel encoding: Security analysis, J. Phys. A: Math. Gen. 35, 10065 (2002).

[22] C. Wang, F. G. Deng, Y. S. Li, X. S. Liu, and G. L. Long, Quantum secure direct communication with high-dimension quantum superdense coding, Phys. Rev. A 71, 044305 (2005).

[23] B. P. Lanyon, M. Barbieri, M. P. Almeida, T. Jennewein, T. C. Ralph, K. J. Resch, G. J. Pryde, J. L. Obrien, A. Gilchrist, and A. G. White, Simplifying quantum logic using higher-dimensional Hilbert spaces, Nat. Phys. 5, 134 (2009).

[24] A. Rossi, G. Vallone, A. Chiuri, F. De Martini, and P. Mataloni, Multipath Entanglement of Two Photons, Phys. Rev. Lett. 102, 153902 (2009).

[25] G. Molina-Terriza, J. P. Torres, and L. Torner, Twisted photons, Nat. Phys. 3, 305 (2007).

[26] L. Neves, S. Pádua, and C. Saavedra, Controlled generation of maximally entangled qudits using twin photons, Phys. Rev. A 69, 042305 (2004).

[27] M. N. O’Sullivan-Hale, I. Ali Khan, R. W. Boyd, and J. C. Howell, Pixel Entanglement: Experimental Realization of Optically Entangled $d=3$ and $d=6$ Qudits, Phys. Rev. Lett. 94, 220501 (2005).

[28] M. A. Solís-Prosser and L. Neves, Remote state preparation of spatial qubits, Phys. Rev. A 84, 012330 (2011).

[29] M. A. Solís-Prosser, M. F. Fernandes, O. Jiménez, A. Delgado, and L. Neves, Experimental Minimum-Error Quantum-State Discrimination in High Dimensions, Phys. Rev. Lett. 118, 100501 (2017).

[30] P. Kolenderski, U. Sinha, L. Youning, T. Zhao, M. Volpini, A. Cabello, R. Laflamme, and T. Jennewein, Aharon-vaidman quantum game with a young-type photonic qutrit, Phys. Rev. A 86, 012321 (2012).

[31] B. Marques, M. R. Barros, W. M. Pimenta, M. A. D. Carvalho, J. Ferraz, R. C. Drumond, M. Terra Cunha, and S. Pádua, Doubleslit implementation of the minimal Deutsch algorithm, Phys. Rev. A 86, 032306 (2012).

[32] S. Etcheverry, G. Caas, E. S. Gmez, W. A. T. Nogueira, C. Saavedra, G. B. Xavier, and G. Lima, Quantum key distribution session with 16-dimensional photonic states, Sci. Rep. 3, 2316 (2013). 
[33] L. Neves, G. Lima, J. G. Aguirre Gómez, C. H. Monken, C. Saavedra, and S. Pádua, Generation of Entangled States of Qudits using Twin Photons, Phys. Rev. Lett. 94, 100501 (2005).

[34] G. Lima, A. Vargas, L. Neves, R. Guzmán, and C. Saavedra, Manipulating spatial qudit states with programmable optical devices, Opt. Express 17, 10688 (2009).

[35] M. A. Solís-Prosser, A. Arias, J. J. M. Varga, L. Rebón, S. Ledesma, C. Iemmi, and L. Neves, Preparing Arbitrary Pure States of Spatial Qudits with a Single Phase-Only Spatial Light Modulator, Opt. Lett. 38, 4762 (2013).

[36] J. J. M. Varga, L. Rebón, M. A. Solís-Prosser, L. Neves, S. Ledesma, and C. Iemmi, Optimized generation of spatial qudits by using a pure phase spatial light modulator, J. Phys. B 47, 225504 (2014).

[37] G. Barreto Lemos, J. O. de Almeida, S. P. Walborn, P. H. Souto Ribeiro, and M. Hor-Meyll, Characterization of a spatial light modulator as a polarization quantum channel, Phys. Rev. A 89, 042119 (2014).

[38] B. Marques, A. A. Matoso, W. M. Pimenta, A. J. GutiérrezEsparza, M. F. Santos, and S. Pádua, Experimental simulation of decoherence in photonics qudits, Sci. Rep. 5, 16049 (2015).

[39] A. V. Oppenheim and J. S. Lim, The importance of phase in signals, Proc. IEEE 69, 529 (1981).
[40] A. Lizana, I. Moreno, A. Márquez, C. Iemmi, E. Fernández, J. Campos, and M. J. Yzuel, Time fluctuations of the phase modulation in a liquid crystal on silicon display: Characterization and effects in diffractive optics, Opt. Express 16, 16711 (2008).

[41] A. Márquez, C. Iemmi, I. Moreno, J. A. Davis, J. Campos, and M. J. Yzuel, Quantitative prediction of the modulation behavior of twisted nematic liquid crystal displays based on a simple physical model, Opt. Eng. 40, 2558 (2001).

[42] A. Márquez, I. Moreno, C. Iemmi, A. Lizana, J. Campos, and M. J. Yzuel, Mueller-stokes characterization and optimization of a liquid crystal on silicon display showing depolarization, Opt. Express 16, 1669 (2008).

[43] A. Fernández-Pérez, A. B. Klimov, and C. Saavedra, Quantum process reconstruction based on mutually unbiased basis, Phys. Rev. A 83, 052332 (2011).

[44] R. Jozsa, Fidelity for mixed quantum states, J. Mod. Opt. 41, 2315 (1994).

[45] See Supplemental Material at http://link.aps.org/supplemental/ 10.1103/PhysRevA.96.032309 for the experimental density matrix evolution in $D=7$ and for a comparison between simulated and experimental density matrix evolution in $D=11$. 